\title{
ON COUNTABLY COMPACT QUASI-PSEUDOMETRIZABLE SPACES
}

\author{
SERGIO SALBANY and SALVADOR ROMAGUERA
}

(Received 15 April 1989; revised 31 October 1989)

Communicated by J. H. Rubinstein

\begin{abstract}
We prove the following results: (1) A quasi-metrizable space is compact if and only if every compatible quasi-metric has a quasi-metric left $d$-sequential completion. (2) A quasipseudometrizable space is countably compact if and only if every compatible quasi-pseudometric is pointwise bounded. (3) A quasi-pseudometrizable space is compact if and only if every compatible quasi-pseudometric is precompact.

1980 Mathematics subject classification (Amer. Math. Soc.) (1985 Revision): 54 D 30, 54 E 35, 54 E 45, 54 E 50, 54 E 52.

Keywords and phrases: left $d$-Cauchy sequence, left $d$-(weakly, sequentially) complete quasipseudometric space, quasi-metric completion, (countably) compact space.
\end{abstract}

\section{Introduction}

It is well-known that for a metrizable space $(X, T)$ the following are equivalent:

(A) $(X, T)$ is (countably) compact;

(B) every metric compatible with $T$ is complete;

(C) every metric compatible with $T$ is (pointwise) bounded;

(D) every metric compatible with $T$ is precompact.

This paper extends these characterizations to quasi-pseudometrizable spaces. However that extension presents some peculiarities. In fact while a countably compact quasi-pseudometrizable space can be characterized by means of conditions of type (B) and (C) (in the "pointwise bounded" case),

(C) 1990 Australian Mathematical Society $0263-6115 / 90 \$ A 2.00+0.00$ 
a compact quasi-pseudometrizable space is characterized by using a condition of type (D). In the light of these facts it seems appropriate to recall that while every countably quasi-metrizable space is compact (see [3, page 40]), compactness and countable compactness are not equivlaent in quasipseudometrizable spaces [2], [5].

Terms and concepts which are not defined are used as in [3]. The letter $\mathbb{N}$ will denote the set of all positive integer numbers.

A quasi-pseudometric on a set $X$ is a non-negative real-valued function $d$ on $X \times X$ such that, for all $x, y, z \in X$ : (i) $d(x, x)=0$; (ii) $d(x, y) \leq$ $d(x, z)+d(z, y)$. If $d$ satisfies the additional condition (iii) $d(x, y)=0$ implies $x=y$, then, $d$ is called a quasi-metric on $X$.

A quasi-(pseudo) metric space is a pair $(X, d)$ such that $X$ is a nonempty set and $d$ is a quasi-(pseudo) metric on $X$.

Each quasi-pseudometric $d$ on $X$ induces a topology $T(d)$ which has as a base the family of $d$-balls $\left\{B_{d}(x, r): x \in X, r>0\right\}$ where $B_{d}(x, r)=$ $\{y \in X: d(x, y)<r\}$. A topological space $(X, T)$ is called quasi-(pseudo) metrizable if there exists a quasi-(pseudo) metric $d$ on $X$ such that $T=$ $T(d)$. In this case we say that $d$ is compatible with $T$.

By using the metrization lemma [4, page 185] the authors have presented [9] a characterization of countably compact quasi-pseudometrizable spaces which extends the Niemytzki-Tychonoff theorem for these spaces. In Section 2 of this paper we shall prove (Theorem 1) a more general result, without using the metrization lemma. In this way we generalize the corresponding result for quasi-metric spaces proved by Fletcher and Lindgren [3, Theorem 7.35] and at the same time provide a simple proof. Furthermore, under very general conditions, it follows that notions of completeness for quasipseudometric spaces which are generally distinct, actually coincide when the topology is countably compact. Our method of proof also permits us to state (Theorem 2) a characterization of compact quasi-metrizable spaces in terms of several kinds of completion. Finally, in Section 3 we prove (Theorem 3) that a quasi-pseudometrizable space is countably compact if and only if every compatible quasi-pseudometric is pointwise bounded and we deduce that a quasi-pseudometrizable space is compact if and only if every compatible quasi-pseudometric is precompact.

\section{Complete quasi-pseudometric spaces and countable compactness}

Intuitively, the following concepts (see [7]) correspond to strongest and weakest notions for a sequence to be "Cauchy". 
Definition 1. Let $(X, d)$ be a quasi-pseudometric space. A sequence $\left\{x_{n}\right\}_{n \in N}$ in $X$ is said to be left $d$-Cauchy if for each $\varepsilon>0$ there are a point $x \in X$ and a $k \in \mathbb{N}$ such that $d\left(x, x_{n}\right)<\varepsilon$ for all $n \geq k$. The sequence $\left\{x_{n}\right\}_{n \in \mathbb{N}}$ is called symmetric $d$-Cauchy ( $d$-Cauchy in [7]) if for each $\varepsilon>0$ there is a $k \in \mathbb{N}$ such that $d\left(x_{n}, x_{m}\right)<\varepsilon$ for all $n, m \geq k$.

Definition 2. We say that a quasi-pseudometric $d$ on a set $X$ is left $d$-sequentially complete if every left $d$-Cauchy sequence in $X$ converges to a point in $X$ (with respect to the topology $T(d)$ ) and we say that $d$ is left $d$-weakly sequentially complete if every left $d$-Cauchy sequence in $X$ has a $T(d)$-cluster point in $X$. Similarly, we say that $d$ is symmetric $d$ sequentially complete if every symmetric $d$-Cauchy sequence in $X$ converges to a point in $X$ (with respect to the topology $T(d)$ ).

A quasi-pseudometric space $(X, d)$ is called left $d$-(weakly) sequentially complete if the quasi-pseudometric $d$ is left $d$-(weakly) sequentially complete. Similarly, $(X, d)$ is called symmetric $d$-sequentially complete if the quasi-pseudometric $d$ is symmetric $d$-sequentially complete.

A filter $\mathscr{F}$ on a quasi-uniform spce $(X, \mathscr{U})$ is called a $\mathscr{U}$-Cauchy filter [3, page 47] if for each $V \in \mathscr{U}$ there is an $x \in X$ such that $V(x) \in \mathscr{F}$. Similarly, the filter $\mathscr{F}$ is called a symmetric $\mathscr{U}$-Cauchy filter if for each $V \in \mathscr{U}$ there is an $F \in \mathscr{F}$ such that $F \times F \subset V$. We say that a quasi-uniform space $(X, \mathscr{U})$ is (symmetric) $\mathscr{U}$-complete if each (symmetric) $\mathscr{U}$-Cauchy filter on $X$ converges to some point in $X$ (with respect to the topology $T(\mathscr{U}))$ and $(X, \mathscr{U})$ is called $\mathscr{U}$-weakly complete if each $\mathscr{U}$-Cauchy filter on $X$ has a $T(\mathscr{U})$-cluster point in $X$.

Definition 3. Let $(X, d)$ be a quasi-pseudometric space and let $\mathscr{U}(d)$ be the quasi-uniformity generated by $d$ (see [3, page 3]). Then $d$ and $(X, d)$ are called left $d$-(weakly) complete if the quasi-uniform space $(X, \mathscr{U}(d))$ is $\mathscr{U}(d)$-(weakly) complete.

The following implications are clearly satisfied for a quasi-pseudometric space $(X, d)$ :

(a) left $d$-(weakly) complete implies left $d$-(weakly) sequentially complete and left $d$-(sequentially) complete implies left $d$-(weakly) sequentially complete.

(b) left $d$-sequentially complete implies symmetric $d$-sequentially complete.

In [6, Examples 1 and 2] H. P. Künzi gives an example of a left $d$-weakly complete quasi-metric space that is not left $d$-sequentially complete and an example of a left $d$-sequentially complete quasi-metric space that is not left $d$-weakly complete. He also gives [6, Example 3] an example of a left $d$ weakly complete and left $d$-sequentially complete quasi-metric space that is not left $d$-complete. Consequently, the converse implications of (a) are not 
satisified. On the other hand it is well-known that every sequence which converges with respect to $T(d)$ is left $d$-Cauchy but, unfortunately, $T(d)$ convergence does not imply symmetric $d$-Cauchyness. However, if $(X, d)$ is symmetric $d$-sequentially complete we have the following equivalences:

LEMMA 1. For a quasi-pseudometrizable space $(X, d)$ the following are equivalent.

(i) $(X, d)$ is symmetric $d$-sequentially complete.

(ii) Every symmetric $d$-Cauchy sequence in $X$ has a $T(d)$-cluster point.

(iii) The quasi-uniform space $(X, \mathscr{U}(d))$ is symmetric $\mathscr{U}(d)$-complete.

(iv) Every symmetric $\mathscr{U}(d)$-Cauchy filter in $X$ has a $T(d)$-cluster point.

Proof. (i) $\Rightarrow$ (ii). Obvious.

(ii) $\Rightarrow$ (i). [7, Theorem 1(iii)].

(iii) $\Rightarrow$ (iv). Obvious.

(iv) $\Rightarrow$ (ii). Obvious.

(i) $\Rightarrow$ (iii). Let $\mathscr{F}$ be a symmetric $\mathscr{U}(d)$-Cauchy filter on $X$. Then there exists a decreasing sequence $\left\{F_{n}\right\}_{n \in \mathbb{N}}$ of subsets of $X$ such that, for all $n \in \mathbb{N}$, we have $F_{n} \in \mathscr{F}$ and $F_{n} \times F_{n} \subset\left\{(x, y): d(x, y)<2^{-n}\right\}$. For each $n \in \mathbb{N}$ choose $x_{n} \in F_{n}$. Then $\left\{x_{n}\right\}_{n \in \mathbb{N}}$ is a symmetric $d$-Cauchy sequence and, thus, it is $T(d)$-convergent to a point $x \in X$. Given $k \in \mathbb{N}$ there exists $n_{0}>k$ such that $d\left(x, x_{n}\right)<2^{-(k+1)}$ for all $n \geq n_{0}$. Hence, for every $y \in F_{k+1}$, we have $d(x, y) \leq d\left(x, x_{n_{0}}\right)+d\left(x_{n_{0}}, y\right)<2^{-(k+1)}+2^{-(k+1)}$ since $x_{n_{0}} \in F_{k+1}$. This implies that $B_{d}\left(x, 2^{-k}\right) \in \mathscr{F}$ and, hence, $\mathscr{F}$ is $T(d)$-convergent to $x$.

In the light of the above lemma we shall use, in the following, the term "symmetric $d$-complete" instead of "symmetric $d$-sequentially complete" for a symmetric $d$-sequentially complete quasi-pseudometric space $(X, d)$.

COROLLARY 1. Every left $d$-weakly sequentially complete quasi-pseudometric space is symmetric d-complete.

Note that [7, Example 4] shows that, in general, the converse implication of (b) is not satisfied.

LeMMA 2. Let $d$ be a quasi-pseudometric on a set $X$ such that $d \leq 1$ and supose that there exists a sequence $\left\{x_{m}\right\}_{m \in \mathbb{N}}$ in $X$ that has no $T(d)$-cluster point in $X$. If for each $n \in \mathbb{N}$ we define $A_{n}=\left\{x_{m}: m \geq n\right\}$ and

$$
e_{n}(x, y)= \begin{cases}d(x, y) & \text { if } x, y \in X-\bar{A}_{n}, \\ 1 & \text { if } x \in X-\bar{A}_{n}, y \in \bar{A}_{n}, \\ 0 & \text { if } x \in \bar{A}_{n},\end{cases}
$$


then the real-valued function $e$, defined on $X \times x$ as

$$
e(x, y)=\sup \left\{2^{-n} e_{n}(x, y): n \in \mathbb{N}\right\},
$$

is a quasi-pseudometric on $X$ such that $T(d)=T(e)$. Moreover, $\left\{x_{m}\right\}_{m \in \mathbb{N}}$ is a symmetric e-Cauchy sequence.

Proof. Since the sequence $\left\{x_{m}\right\}_{m \in \mathbb{N}}$ has no $T(d)$-cluster point, we deduce that $X=\bigcup_{n=1}^{\infty}\left(X-\bar{A}_{n}\right)$. It is straightforward to verify that each $e_{n}$ satisfies the triangle inequality and, therefore, $e$ is a quasi-pseudometric on $X$. Assume $\left\{y_{m}\right\}_{m \in \mathbb{N}}$ is a $T(e)$-convergent sequence to a point $y \in X$. Then, for each $n \in \mathbb{N}, e_{n}\left(y, y_{m}\right) \rightarrow 0$ as $m \rightarrow \infty$. For the given $y$, let $k$ be the smallest index for which $y \in X-\bar{A}_{k}$. Then, $e_{k}\left(y, y_{m}\right) \rightarrow 0$ gives $e_{k}\left(y, y_{m}\right)<1$ eventually, so that $\left\{y_{m}\right\}_{m \in \mathbf{N}}$ is eventually in $X-\bar{A}_{k}$ by definition of $\boldsymbol{e}_{k}$. Hence, there is $j \in \mathbb{N}$ such that, if $m \geq j, d\left(y, y_{m}\right)=e_{k}\left(y, y_{m}\right)$. Thus, $\left\{y_{m}\right\}_{m \in N}$ is $T(d)$-convergent to $y$. Conversely, assume $\left\{y_{m}\right\}_{m \in \mathbb{N}}$ is $T(d)$ convergent to $y$. For the given $y$ let $k$ be the smallest index for which $y \in X-\bar{A}_{k}$. Then, there is $j \in N$ such that, if $m \geq j, e_{n}\left(y, y_{m}\right)=d\left(y, y_{m}\right)$ for all $n \geq k$. Consequently, $e\left(y, y_{m}\right)=2^{-k} d\left(y, y_{m}\right)$ for $m \geq j$. This proves that $\left\{y_{m}\right\}_{m \in \mathrm{N}}$ is $T(e)$-convergent to $y$ and, hence, $T(e)=T(d)$.

Finally, $\left\{x_{m}\right\}_{m \in \mathbb{N}}$ is a symmetric $e$-Cauchy sequence since given $\varepsilon>0$ let $j \in \mathbb{N}$ be such that $2^{j} \varepsilon>1$. Let $n, m \geq j$. Then, for $k=1,2, \ldots, j$, we have $e_{k}\left(x_{n}, x_{m}\right)=0$ since $x_{n}, x_{m} \in A_{k}$. For $k>j, e_{k}\left(x_{n}, x_{m}\right) \leq 1$ so that, for $n, m \geq j, e\left(x_{n}, x_{m}\right)<2^{-j}<\varepsilon$.

THEOREM 1. For a quasi-pseudometrizable space $(X, T)$ the following are equivalent.

(i) $(X, T)$ is countably compact.

(ii) Every quasi-pseudometric $d$ on $X$ compatible with $T$ is left $d$-complete.

(iii) Every quasi-pseudometric $d$ on $X$ compatible with $T$ is left $d$-weakly sequentially complete.

(iv) Every quasi-pseudometric $d$ on $X$ compatible with $T$ is symmetric d-complete.

Proof. (i) $\Rightarrow$ (ii). [6, Proposition 5].

(ii) $\Rightarrow$ (iii). Obvious.

(iii) $\Rightarrow$ (iv). Corollary 1 .

(iv) $\Rightarrow$ (i). Suppose that there exists a sequence $\left\{x_{n}\right\}_{n \in N}$ in $X$ that has no $T$-cluster point. Let $d$ be a quasi-pseudometric on $X$ compatible with $T$ satisfying $d \leq 1$. By Lemma 2 , there exists a quasi-pseudometric $e$ on $X$ compatible with $T$ such that $\left\{x_{m}\right\}_{m \in \mathbb{N}}$ is a symmetric $e$-Cauchy 
sequence. Hence, the quasi-pseudometric $e$ is not symmetric $e$-complete. This concludes the proof.

CoRollary [3, page 178]. A quasi-metrizable space $(X, T)$ is compact if, and only if, every compatible quasi-metric $d$ is left $d$-weakly (sequentially) complete.

Let $(X, \mathscr{U})$ be a $T_{1}$ quasi-uniform space. A quasi-uniform space $(Y, \mathscr{V})$ is called a (weak) $\mathscr{U}$-completion of $(X, \mathscr{U})$ if $(Y, \mathscr{V})$ is a $\mathscr{V}$-(weakly) complete $T_{1}$ quasi-uniform space that has a dense subspace quasi-unimorphic (relative to $\mathscr{U}$ and $\mathscr{V}$ ) to $(X, \mathscr{U})$ [3]. The concept of symmetric completion is defined in the analogous manner where $(Y, \mathscr{V})$ is symmetric $\mathscr{V}$-complete.

Let $(X, d)$ be a quasi-metric subspace of a quasi-metric space $(\hat{X}, \hat{d})$. We say that $(\hat{X}, \hat{d})$ is a quasi-metric left $d$-(weak) sequential completion of $(X, d)$ if $X$ is dense in $(\hat{X}, T(\hat{d}))$ and $(\hat{X}, \hat{d})$ is left $\hat{d}$-(weakly) sequentially complete. The concepts of quasi-metric left $d$-completion, quasi-metric left $d$-weak completion and quasi-metric symmetric $d$-completion are defined in the obvious manner where $(\hat{X}, \hat{d})$ is left $\hat{d}$-complete, left $\hat{d}$-weakly complete and symmetric $\hat{d}$-complete, respectively.

It is interesting to recall that a Hausdorff quasi-metric space need not have a quasi-metric symmetric $d$-completion [8, Theorem 2.6] (a similar situation occurs for quasi-uniform spaces [1], [3]).

In [3, Theorem 3.43 and Proposition 3.46] are proved the two following results.

(1) A $T_{1}$ quasi-uniform space $(X, \mathscr{U})$ has a weak $\mathscr{U}$-completion if and only if whenever $\mathscr{F}$ is a $\mathscr{U}$-Cauchy filter on $X$ and $x \in X$ is a $T\left(\mathscr{U}^{-1}\right)$ cluster point of $\mathscr{F}$, then $x$ is a $T(\mathscr{U})$-cluster point of $\mathscr{F}$.

(2) A $T_{1}$ topological space $(X, T)$ is compact if and only if every quasiuniformity $\mathscr{U}$ on $X$ compatible with $T$ has a weak $\mathscr{U}$-completion.

In [6, Proposition 8] H. P. Künzi obtains an analogue of (1) for quasimetric spaces. The following result provides an analogue of $(2)$ for quasimetric spaces.

THEOREM 2. For a quasi-metrizable space $(X, T)$ the following are equivalent.

(i) $(X, T)$ is compact.

(ii) Every quasi-metric $d$ on $X$ compatible with $T$ has a quasi-metric left d-completion.

(iii) Every quasi-metric $d$ on $X$ compatible with $T$ has a quasi-metric left $d$-weak sequential completion. 
(iv) Every quasi-metric $d$ on $X$ compatible with $T$ has a quasi-metric symmetric d-completion.

Proof. (i) $\Rightarrow$ (ii). Theorem 1.

(ii) $\Rightarrow$ (iii). Obvious.

(iii) $\Rightarrow$ (iv). Obvious.

(iv) $\Rightarrow$ (i). Suppose that $(X, T)$ is not compact. Then there exists a sequence $\left\{x_{m}\right\}_{m \in \mathbf{N}}$ in $X$ that has no cluster point. Let $d$ be a quasi-metric compatible with $T$ such that $d \leq 1$. Consider the quasi-pseudometric $e$ constructed in Lemma 2 and note that, in this case, $e$ is a quasi-metric. Moreover $T(e)=T$ and $\left\{x_{m}\right\}_{m \in \mathbf{N}}$ is a symmetric $e$-Cauchy sequence. We shall show that, for each $x \in X, e\left(x_{m}, x\right) \rightarrow 0$. Let $k$ be the smallest index for which $x \in X-\bar{A}_{k}$. Therefore, for $n \geq k, e_{n}\left(x_{m}, x\right)=d\left(x_{m}, x\right)$ when $x_{m} \in X-\bar{A}_{n}$, and $e_{n}\left(x_{m}, x\right)=0$ when $x_{m} \in \bar{A}_{n}$. Given $\varepsilon>0$ let ' $j>k$ be such that $2^{j} \varepsilon>1$. Hence, for $m \geq j, e\left(x_{m}, x\right) \leq 2^{-(j+1)}<\varepsilon$. Assume $(\hat{X}, \hat{e})$ is a quasi-metric symmetric $e$-completion of $(X, e)$. Then $\left\{x_{m}\right\}_{m \in \mathrm{N}}$ is a symmetric $\hat{e}$-Cauchy sequence and, therefore, there is a point $p \in \hat{X}$ satisfying $\hat{e}\left(p, x_{m}\right) \rightarrow 0$. Since $\hat{e}\left(x_{m}, x\right) \rightarrow 0$ we deduce $p=x \in X$ because $\hat{e}$ is a quasi-metric. This implies $e\left(x, x_{m}\right) \rightarrow 0$ and, consequently, the sequence $\left\{x_{m}\right\}_{m \in \mathrm{N}}$ is $T(e)$-convergent to $x$, a contradiction. The proof is complete.

We conclude this section with some remarks concerning different notions of completion of a quasi-metric space.

We first note that in the above theorems the conditions 'left $d-$ ', left $d$ weak(ly)' and 'symmetric $d$ ' can be replaced by other notions of completenss in the sense of [7]. For instance, it follows immediately from Theorem 2 that a quasi-metrizable space $(X, T)$ is compact if and only if every quasi-metric $d$ on $X$ compatible with $T$ has a quasi-metric left $K$-completion (see [7, pages 132 and 134]). On the other hand, H. P. Künzi [6] has shown that every quasi-metric space having a quasi-metric left $d$-weak sequential completion has a base of countable order. His method of proof actually shows a more general result, namely, every quasi-metric space having a quasi-metric left $K$-completion has a base of countable order. It follows from this observation that the Sorgenfrey line is a quasi-metric space that does not admit compatible quasi-metrics that have a quasi-metric left $K$-completion because this space does not have a base of countable order.

In [6] Künzi has also given an example of a quasi-metric space $(X, d)$ that has a quasi-metric left $d$-weak sequential completion but does not have a quasi-metric left $d$-weak completion. In order to complete this jigsaw puzzle we give here a simple example of a left $d$-weakly complete quasi-metric space 
that does not admit any quasi-metric left $d$-sequential completion.

EXAMPLE 1 Let $X$ be the set of all non-negative integer numbers and define a quasi-metric $d$ on $X$ as follows.

$$
\begin{array}{ll}
d(0, x)=1 & \text { if } x \neq 0, \\
d(1, x)=1 & \text { if } x \neq 1, \\
d(n, 0)=d(n, 1)=1 / n & \text { if } n \in X-\{0,1\}, \\
d(n, x)=1 & \text { if } n \neq x \text { and } n, x \in X-\{0,1\}, \\
d(x, x)=0 & \text { for all } x \in X .
\end{array}
$$

Then $T(d)$ is the discrete topology on $X$. The sequence $\left\{x_{m}\right\}_{m \in N}$ such that $x_{2 n}=0$ and $x_{2 n+1}=1$ for all $n \in \mathbb{N}$, is a left $d$-Cauchy sequence that is not $T(d)$-convergent. Assume $(\hat{X}, \hat{d})$ is a quasi-metric left $d$-sequential completion of $(X, d)$. Then $\hat{d}\left(p, x_{n}\right) \rightarrow 0$ for some $p \in \hat{X}$ and, hence, $p=0$ and $p=1$, a contradiction. On the other hand it is clear that every $\mathscr{U}(d)$-Cauchy filter on $X$ has a $T(d)$-cluster point.

\section{Pointwise bounded quasi-pseudometrics and countable compactness}

A quasi-pseudometric $d$ on a set $X$ is called bounded if there is constant $M>0$ such that $d(x, y) \leq M$ for every $x, y \in X$.

The following example shows that there exists a quasi-metric space $(X, d)$ such that $(X, T(d))$ is a compact (and metrizable) space but $d$ is not bounded.

EXAMPLE 2. Let $X$ be the set of all non-negative integer numbers and. define a quasi-metric $d$ on $X$ as follows:

$$
\begin{array}{ll}
d(0, x)=1 / x & \text { for all } x \in X-\{0\}, \\
d(x, y)=x & \text { for all } x \neq 0 \text { and } y \neq x, \\
d(x, x)=0 & \text { for all } x \in X .
\end{array}
$$

It is obvious that $d$ is not bounded. However $T(d)$ is a Hausdorff compact space and hence it is metrizable. Note that, still, given $x \in X$ there exists a constant $M_{x}>0$ such that, for all $y \in X, d(x, y) \leq M_{x}$. This fact suggests the next definition.

Definition 4. We say that a quasi-pseudometric $d$ on a set $X$ is pointwise bounded if for some $x \in X$ there exists a constant $M_{x}>0$ such that, for all $y \in X, d(x, y) \leq M_{x}$.

It is easy to see that boundedness and pointwise boundedness are equivalent for a pseudometric. 
Lemma 3. Let $d$ be a quasi-pseudometric on a set $X$ such that $d \leq 1$ and suppose that there exists a sequence $\left\{x_{m}\right\}_{m \in \mathbf{N}}$ in $X$ that has no $T(d)$-cluster point in $X$. If for each $n \in \mathbb{N}$ we define $A_{n}=\left\{x_{m}: m \geq n\right\}$ and

$$
e_{n}(x, y)= \begin{cases}d(x, y) & \text { if } x, y \in X-\bar{A}_{n}, \\ 2^{2 n} & \text { if } x \in X-\bar{A}_{n}, y \in \bar{A}_{n}, \\ 0 & \text { if } x \in \bar{A}_{n},\end{cases}
$$

then the real-valued function $e$, defined on $X \times X$ as

$$
e(x, y)=\sup \left\{2^{-n} e_{n}(x, y): n \in \mathbb{N}\right\},
$$

is a quasi-pseudometric on $X$ such that $T(d)=T(e)$. Moreover, $e$ is not pointwise bounded.

Proof. A slight modification of the proof of Lemma 2 permits us to conclude that $e$ is a quasi-pseudometric on $X$ compatible with $T(d)$. Now let $x \in X$ and let $k$ be the smallest index for which $x \in X-\bar{A}_{k}$. For each $m \geq k$ we have $e_{m}\left(x, x_{m}\right)=2^{2 m}$ and, hence, $e\left(x, x_{m}\right) \geq 2^{m}$ for $m \geq k$. Thus $e$ is not pointwise bounded.

Theorem 3. A quasi-pseudometrizable space $(X, T)$ is countably compact if and only if every quasi-pseudometric on $X$ compatible with $T$ is pointwise bounded.

Proof. Let $(X, T)$ be a countably compact quasi-pseudometrizable space and let $d$ be a compatible quasi-pseudometric on $X$. Suppose that there are a point $x \in X$ and a sequence $\left\{x_{m}\right\}_{m \in \mathbb{N}}$ in $X$ satisfying $d\left(x, x_{m}\right) \geq m$ for all $m \in \mathbb{N}$. Since $\left\{x_{m}\right\}_{m \in \mathbb{N}}$ has a subsequence $\left\{x_{m(k)}\right\}_{k \in \mathbb{N}}$ which is $T(d)$ convergent to some pont $y \in X$, we obtain, from $d\left(x, x_{m(k)}\right) \leq d(x, y)+$ $d\left(y, x_{m(k)}\right)$, a contradiction. The converse follows immediately from Lemma 3.

A quasi-pseudometric on a set $X$ is called precompact if for each $\varepsilon>0$ there exists a finite subset $F$ of $X$ such that $d(F, x)<\varepsilon$ for all $x \in X$.

We omit the easy proof of the following observation

LEMMA 4. Every precompact quasi-pseudometric is pointwise bounded.

Theorem 4. A quasi-pseudometrizable space $(X, T)$ is compact if and only if every quasi-pseudometric on $X$ compatible with $T$ is precompact.

Proof. Suppose that every quasi-pseudometric compatible with $T$ is precompact. By Lemma 4 and Theorem $4,(X, T)$ is countably compact. 
Choose a compatible quasi-pseudometric $d$ on $X$. By Theorem $1, d$ is left $d$-weakly complete and, therefore, the quasi-uniform space $(X, \mathscr{U}(d))$ is precompact and $\mathscr{U}(d)$-weakly complete. It follows from [3, Theorem 3.24] that $(X, T)$ is compact. The converse is well-known.

\section{Acknowledgements}

The authors thank the referee for his valuable suggestions. We also acknowledge the privilege of having seen [6] prior to publication.

\section{References}

[1] J. W. Carlson and T. L. Hicks, 'On completeness in a quasi-uniform space', J. Math. Anal. Appl. 34 (1971), 618-627.

[2] J. Ferrer and V. Gregori, 'A sequentially compact non-compact quasi-pseudometric space', Monatsh. Math. 96 (1983), 269-270.

[3] P. Fletcher and W. F. Lindgren, Quasi-uniform spaces, (Marcel Dekker, New York, 1982).

[4] J. L. Kelley, General topology, (Springer-Verlag, New York, 1955).

[5] H. P. Künzi, 'A note on sequentially compact quasi-pseudo-metric spaces', Monatsh. Math. 95 (1983), 219-220.

[6] H. P. Künzi, 'Complete quasi-pseudo-metric spaces', to appear.

[7] I. L. Reilly, P. V. Subrahmanyam and M. K. Vamanamurthy, 'Cauchy sequences in quasi-pseudo-metric spaces', Monatsh. Math. 93 (1982), 127-140.

[8] S. Romaguera and A. Gutiérrez, 'A note on Cauchy sequences in quasi-pseudo-metric spaces', Glas. Mat. 21 (1986), 191-200.

[9] S. Romaguera and S. Salbany, 'On the Niemytzki-Tychonoff theorem for distance spaces', to appear.

Department of Mathematics

University of Zimbabwe

P. O. Box MP 167

Mount Pleasant, Harare

Zimbabwe
Departamento de Matemática

Aplicada

Escuela de Caminos

Universidad Politécnica

46071 Valencia

Spain 